\title{
Ultrafast Spin Dynamics in Multisublattice Magnets
}

\author{
J. H. Mentink, ${ }^{1, *}$ J. Hellsvik, ${ }^{2,3}$ D. V. Afanasiev, ${ }^{4}$ B. A. Ivanov, ${ }^{4}$ A. Kirilyuk, ${ }^{1}$ A. V. Kimel, ${ }^{1}$ O. Eriksson, ${ }^{2}$ \\ M. I. Katsnelson, ${ }^{1}$ and Th. Rasing ${ }^{1}$ \\ ${ }^{1}$ Radboud University Nijmegen, Institute of Molecules and Materials, Heyendaalseweg 135, 6525 AJ Nijmegen, The Netherlands \\ ${ }^{2}$ Department of Physics and Astronomy, Uppsala University, Box 516, SE-751 20, Uppsala, Sweden \\ ${ }^{3}$ Consiglio Nazionale delle Ricerche-Superconducting and Innovative Materials and Devices (CNR-SPIN), 67100 L'Aquila, Italy \\ ${ }^{4}$ Institute of Magnetism, NASU, 03142 Kiev, Ukraine \\ (Received 18 May 2011; published 30 January 2012)
}

\begin{abstract}
We propose a general theoretical framework for ultrafast laser-induced spin dynamics in multisublattice magnets. We distinguish relaxation of relativistic and exchange origin and show that when the former dominates, nonequivalent sublattices have distinct dynamics despite their strong exchange coupling. Even more interesting, in the exchange dominated regime sublattices can show highly counterintuitive transitions between parallel and antiparallel alignment. This allows us to explain recent experiments with antiferromagnetically coupled sublattices, and predict that such transitions are possible with ferromagnetic coupling as well. In addition, we predict that exchange relaxation enhances the demagnetization speed of both sublattices only when they are antiferromagnetically coupled.
\end{abstract}

DOI: 10.1103/PhysRevLett.108.057202

PACS numbers: 75.78.Jp, 75.40.Gb, 75.40.Mg

Intense femtosecond laser pulses are able to excite and transform magnetic order of condensed matter systems on a time scale of the exchange interaction [1], i.e., on a time scale pertinent to the period of spin motion in the exchange field. Laser-induced demagnetization [2], magnetization reversal [3], change of magnetic anisotropy [4] or even a change of the exchange integral [5] have been experimentally demonstrated. These observations have pushed spin dynamics to a new regime, where the observed dynamics appears to be dominantly longitudinal, which can thus not be described by the conventional transverse LandauLifshitz dynamics [6] which conserves the magnitude of the angular momentum for any sublattice. Although many experiments have been done in magnets with more than one magnetic sublattice, this fact has largely been ignored in the theoretical descriptions for longitudinal spin dynamics [7-10]. This suggests that in the experiments the different sublattices have the same dynamics. In striking contrast, femtosecond $\mathrm{x}$-ray probes $[11,12]$ have elucidated recently that the sublattices in ferrimagnetic GdFe have distinct dynamics after femtosecond laser excitation [13], and this has been observed more recently also in permalloy [14].

In this Letter we present a general theoretical framework for ultrafast spin dynamics in multisublattice magnets which contains longitudinal relaxation of both relativistic and exchange origin. The latter is the key new ingredient of our theory and is only present in magnets with more than one sublattice. The reason is simply that the exchange

Published by the American Physical Society under the terms of the Creative Commons Attribution 3.0 License. Further distribution of this work must maintain attribution to the author(s) and the published article's title, journal citation, and DOI. interaction conserves the total angular momentum and therefore longitudinal exchange relaxation in magnets with only one sublattice is not possible. In the spirit of the models for one sublattice [8-10] we consider multisublattice magnets coupled to a heat bath with a (time dependent) temperature $T$. The heat bath represents the environment and can often be taken as the electron system, which rapidly heats upon laser excitation and subsequently cools down due to the coupling with the lattice, as can be modeled conveniently by a two-temperature model [15]. The magnetic system is considered to be neither in equilibrium with itself nor with the heat bath, as we are interested in the relaxation of the magnetic sublattices in approach to equilibrium. In order to illustrate our theory we classify the dynamics that occur in multisublattice magnets in three regimes, depending on whether the temperature of the heat bath is above, below, or in the vicinity of the critical temperature $T_{C}$ of the multisublattice magnet. We illustrate our theory by showing explicit solutions in the regime $T<T_{C}$. Finally, we substantiate our theory with recently reported experiments [13] and by performing atomistic spin dynamics simulations.

The basis of our theoretical framework is the description of spin dynamics using Onsager's relations. Such an approach was first employed by Baryakhtar [16], who showed that using Onsager's relations and accounting for the symmetry of the exchange interaction naturally yields dynamics of the length of the macroscopic sublattice magnetizations, pertinent to the time scale of the exchange interaction. On this time scale the conventional transverse dynamics of the angular momentum is negligible and we can limit ourselves to longitudinal dynamics, such that the equations of motion for two nonequivalent collinear sublattices can be written as: 


$$
\begin{gathered}
\dot{S}_{1}=\lambda_{e}\left(H_{1}-H_{2}\right)+\lambda_{1} H_{1}, \\
\dot{S}_{2}=-\lambda_{e}\left(H_{1}-H_{2}\right)+\lambda_{2} H_{2} .
\end{gathered}
$$

Here, $\lambda_{i}$ is of relativistic origin and describes transfer of angular momentum between sublattice $i=1,2$ and the environment. $\lambda_{e}$ is of exchange origin and stems from spinspin interactions, conserving the total angular momentum but allowing for the transfer of angular momentum between the sublattices. $S_{i}$, which can be both positive and negative, denotes the macroscopic angular momentum of sublattice $i$ and is related to the magnetization $M_{i}$ by the gyromagnetic ratio $\gamma_{i}: S_{i}=M_{i} / \gamma_{i}$. Further $\dot{S}_{i}=d S_{i} / d t$ and the effective fields $H_{i}=-\delta W / \delta S_{i}$ are derived from the magnetic energy $W$. In the uniform exchange approximation $W$ can be written as [16]

$$
W=\int d \mathbf{x}\left\{f_{1}\left(S_{1}^{2}\right)+f_{2}\left(S_{2}^{2}\right)+f_{12}\left(S_{1} S_{2}\right)\right\} .
$$

Here, $f_{i}\left(S_{i}^{2}\right)$ determines the exchange energy responsible for the formation of the macroscopic magnetic moment in sublattice $i . f_{12}=-J_{12} S_{1} S_{2}$ is the exchange interaction between the sublattices, determining their mutual orientation, where $J_{12}>0$ for ferromagnetic (FM) coupling and $J_{12}<0$ for antiferromagnetic (AFM) coupling. Generally the exchange energies $f_{i}$ and $J_{12}$ in $W$ are parametrically dependent on the temperature of the environment. We use this to classify the dynamics in three regimes.

The first regime, which we call the temperature dominated regime, is defined as $T \gg T_{C}$. In this regime the system responds as if it was a paramagnet and we may write $f_{i}=S_{i}^{2} /\left(2 \chi_{i}\right)$, where $\chi_{i} \sim 1 / T$ denotes the longitudinal susceptibility of sublattice $i$. Since in the paramagnetic regime $J_{12} \ll k_{B} T$, the interaction between sublattices can be neglected, and the transfer of angular momentum with the environment dominates the dynamics. Consequently, the sublattices exhibit Bloch relaxation, with a relaxation time $\tau_{i}=\chi_{i} / \lambda_{i}$. Microscopic calculations $[17,18]$, show that the longitudinal relaxation time can be written as

$$
\tau_{i}=\mu_{i} /\left(2 \alpha_{i} \gamma k_{B} T\right)
$$

where $\alpha_{i}$ is a microscopic parameter of relativistic origin determining the coupling with the heat bath. Importantly, it follows that the longitudinal relaxation is determined by the atomic magnetic moment $\mu_{i}$, which is intuitively easy to understand. Reducing $S_{i}$ requires transfer of angular momentum, which is limited by the value of $\alpha_{i}$. Therefore, systems with small magnetic moments relax faster. Interestingly, this analysis shows that sublattices with different magnetic moments generally show distinct dynamics in the temperature dominated regime, despite their strong exchange coupling in the ground state. This regime can be accessed by suddenly heating the electron system using a fs laser pulse. Then, on the time scale of
10-100 fs, the electron temperature will rise far above the Curie temperature, such that the spins feel a very hot environment. Such distinct dynamics has recently been observed using fs XMCD probes on fs laser excited ferrimagnetic GdFeCo [13].

The second regime, which we call the exchange dominated regime, is determined by $T<T_{C}$. Since in the ordered regime generally exchange interactions are stronger than relativistic interactions, this regime is characterized by $\lambda_{i} \ll \lambda_{e}$ and generally appears on the ps time scale. In this regime the transfer of angular momentum between the sublattices dominates the dynamics. For purely exchange driven dynamics the total angular momentum is conserved. As a consequence, for any form of the free energy $W$, the changes of the sublattice angular momentum sum up (approximately) to zero: $\dot{S}_{1}=-\dot{S}_{2}$. This yields highly counter-intuitive dynamics when the spin of one of the sublattices is close to zero. Contrasting the temperature dominated regime which would yield $\dot{S}_{i} \rightarrow 0$ when $S_{i} \rightarrow 0$, in the exchange dominated regime $\dot{S}_{i}$ remains finite even when $S_{i}=0$. Therefore, the spin of the sublattices can reverse purely driven by the exchange relaxation. To illustrate this we consider $S_{2}<0$ approaching zero while $S_{1}>0$. We can express the requirement for sublattice reversal to occur with AFM coupling as

$$
\left.\dot{S}_{2}\right|_{S_{2}=0}>0 \Leftrightarrow 2 \partial f_{1} / \partial S_{1}^{2}>-J_{12}\left(1+\lambda_{2} / \lambda_{e}\right)>0 .
$$

Since $J_{12}<0$ it is required that $\partial f_{1} / \partial S_{1}^{2}>0$. By definition $f_{i}\left(S_{i}^{2}\right)$ has a minimum at $S_{i}^{2}=\bar{S}_{i}^{2}$, where $\bar{S}_{i}^{2}$ is the equilibrium value of the angular momentum at the specified temperature. Consequently, to fulfill requirement (5) we need $S_{1}^{2} \gg \bar{S}_{1}^{2}$, which illustrates that sublattice reversal can only occur under strongly nonequilibrium conditions. This inequality can be satisfied when the temperature of the heat bath suddenly increases, as appears after fs laser excitation of the electron system, such that $f_{1}$ dominates over $f_{12}$ and $S_{1}$ lags behind the excitation.

In order to illustrate this novel exchange driven dynamics further we solve the Eq. (1) and (2) at constant temperature of the heat bath. For a typical rare-earth $(i=1)$ transition-metal $(i=2)$ ferrimagnet we model the free energy in the Landau form as $f_{1}=A S_{1}^{2} / 2, f_{2}=$ $B\left(S_{2}^{2}-\bar{S}_{2}^{2}\right)^{2} / 4$, where we assume the usual FM form for sublattice 2 while we take $S_{1}$ to be paramagnetic. For the present simulation we used the values $A / B=0.4, B=$ $1=\bar{S}_{2}, \quad J_{12} / B=-0.15, \quad \lambda_{1}=\lambda_{2}=0.15$ and $\lambda_{e}=1$. The result of the simulations is shown in Fig. 1 by plotting the dynamics of $S_{1}$ as function of $S_{2}$ for various initial conditions. In this phase plane, a pure exchange relaxation appears as trajectories at $45^{\circ}$ from the vertical axis, fulfilling $\dot{S}_{1} / \dot{S}_{2}=-1$. Such trajectories occupy the majority of the phase plane, bounded by lines of partial equilibrium along which slow dynamics owing to pure relativistic relaxation dominates. The thick dots indicate stable equilibria. For our parameters the origin is a saddle point and 


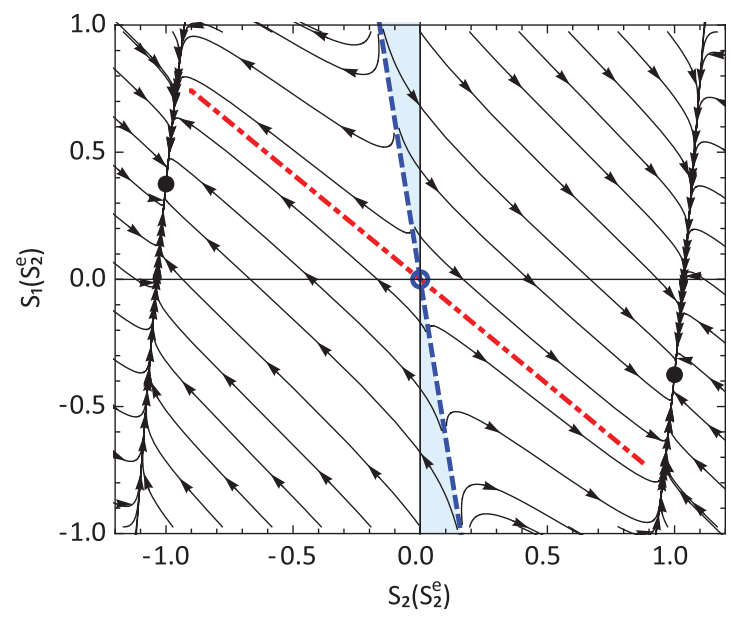

FIG. 1 (color online). Numerical solution of the longitudinal equations of motion in the exchange dominated regime. The evolution of $S_{1}$ is shown as function of $S_{2}$, where both are normalized to the final value $S_{2}^{e}$, for various initial conditions. The arrows indicate the direction when time increases. The thick dots indicate stable equilibrium points. The origin is a saddle point with red and blue (dot)dashed lines indicating (un)stable manifolds. The blue shaded area encompasses the initial conditions from which the longitudinal relaxation will proceed to reversal showing temporal ferromagnetic alignment.

the red and blue (dot)dashed lines indicate (un)stable manifolds. The blue shaded area, bounded by the stable manifold and the vertical axis defines a region of nonequilibrium conditions where $\left|S_{2}\right|<S_{1}$ and $S_{2}$ is substantially demagnetized. The simulations show that when the system is brought into this regime, it will proceed to reverse the sublattices showing temporal ferromagnetic alignment. Using the Landau expansion criterion Eq. (5) gives $\lambda_{2}<$ $\lambda_{e}\left(A /\left|J_{12}\right|-1\right)$. Since by definition $\lambda_{2} \geq 0$, we find that reversal is only possible when exchange relaxation is included $\left(\lambda_{e}>0\right)$. We stress that this type of reversal, driven by a temperature increase and without any magnetic field has recently been discovered [19].

Similarly, we can express the requirement for sublattice reversal to occur for FM coupling as

$$
\left.\dot{S}_{2}\right|_{S_{2}=0}<0 \Leftrightarrow 2 \partial f_{1} / \partial S_{1}^{2}<-J_{12}\left(1+\lambda_{2} / \lambda_{e}\right)<0 .
$$

Since $J_{12}>0$ it is required that $S_{1}^{2} \ll \bar{S}_{1}^{2}$ for the reversal of $S_{2}$. Note that criterion (6) requires a specific form of the energy $f_{1}\left(S_{1}^{2}\right)$, i.e., it cannot appear in a paramagnetic sublattice where $\bar{S}_{1}^{2}=0$, while it does not require a specific form for the energy $f_{2}\left(S_{2}^{2}\right)$. We predict that, contrary to the case with AFM coupling, sublattice reversal in FM coupled sublattices does not occur after a sudden increase of the electron temperature. Instead, requirement (6) typically occurs after a large and sudden decrease of the temperature. We anticipate this effect in materials exhibiting a strong magnetocalorimetric effect [20].
We can also define the criterion for reversal of the total angular momentum $S=S_{1}+S_{2}$ :

$$
\begin{aligned}
\left.\dot{S}\right|_{S=0}<0 \Leftrightarrow- & 2 S_{1}\left(\lambda_{1} \partial f_{1} / \partial S_{1}^{2}-\lambda_{2} \partial f_{2} / \partial S_{2}^{2}\right. \\
& \left.+J_{12}\left(\lambda_{1}-\lambda_{2}\right) / 2\right)<0 .
\end{aligned}
$$

Note that Eq. (7) only contains relaxation terms of relativistic origin as $\lambda_{e}$ conserves $S$.

The third regime, which we call the critical regime, is determined by a temperature close to the critical temperature $T \sim T_{C}$. Here both relativistic and exchange relaxation are of importance and this case is highly relevant for ultrafast demagnetization. To simplify the analysis we neglect the intersublattice contribution to the effective fields and write

$$
\dot{S}_{1}=-\lambda_{1} S_{1} / \chi_{1}-\lambda_{e}\left(S_{1} / \chi_{1}-S_{2} / \chi_{2}\right),
$$

with a similar equation for $S_{2}$, where we assumed $f_{i}=$ $S_{i}^{2} /\left(2 \chi_{i}\right)$ since in the vicinity of $T_{C} \bar{S}_{i} \approx 0$. From Eq. (8) it follows that the effect of exchange relaxation is quite different in AFM and FM coupled sublattices. When the sublattices are antiparallel, we have $S_{1} / \chi_{1}-S_{2} / \chi_{2}=$ $S_{1} / \chi_{1}+\left|S_{2}\right| / \chi_{2}>0$, and hence we predict that both sublattices demagnetize faster than in the uncoupled case $\lambda_{e}=0$. With parallel sublattices either $S_{1}$ or $S_{2}$ is accelerated while the other is decelerated depending on whether $S_{1} / \chi_{1}>S_{2} / \chi_{2}$. Hence relativistic and exchange relaxation can have different sign, which may have direct consequences for the demagnetization of ferromagnetic alloys like FeNi or FeCo. Similar conclusions are obtained when $J_{12}$ is included in the analysis, at least when $k_{B} T_{C} \gg\left|J_{12}\right|$.

To further substantiate the applicability of our theory we employ atomistic spin dynamics (ASD) simulations, using the UppASD method [21,22]. Such simulations solve the dynamics of exchange coupled atomic spins coupled to a heat bath and hence goes beyond the conventional LandauLifshitz dynamics for macrospins. The heat bath ensures longitudinal dynamics of individual sublattices, while the coupling between atomic spins of different sublattices allows for exchange relaxation. We take a ferrimagnetic GdFe model system based on the cubic Laves phase structure characterized by the microscopic exchange parameters $J_{11}=0.2 \mathrm{meV}, J_{12}=-2 \mathrm{meV}, J_{22}=20 \mathrm{meV}$ for nearest neighbor interactions only. For the magnetic moments we take the bulk values $\mu_{1}=7.6 \mu_{B}(\mathrm{Gd})$ and $\mu_{2}=$ $2.1 \mu_{B}(\mathrm{Fe})$. As system size we chose $D=20^{3}$ unit cells and we averaged the simulations over $N=10$ realizations of the heat bath. For these parameters we get $T_{C}=800 \mathrm{~K}$ and a compensation temperature of about $T_{A}=300 \mathrm{~K}$ (data not shown). The coupling to the thermal bath is fixed by $\alpha_{1}=\alpha_{2}=0.02$, and we also used equal gyromagnetic ratios. Following [10], we model laser-induced spin dynamics by a time dependent temperature of the heat bath, which for simplicity is described by a rise time $\tau_{1}=10 \mathrm{fs}$ and a relaxation time $\tau_{2}=1 \mathrm{ps}$, capturing the essential physics of a two-temperature model [15]. The heat bath 


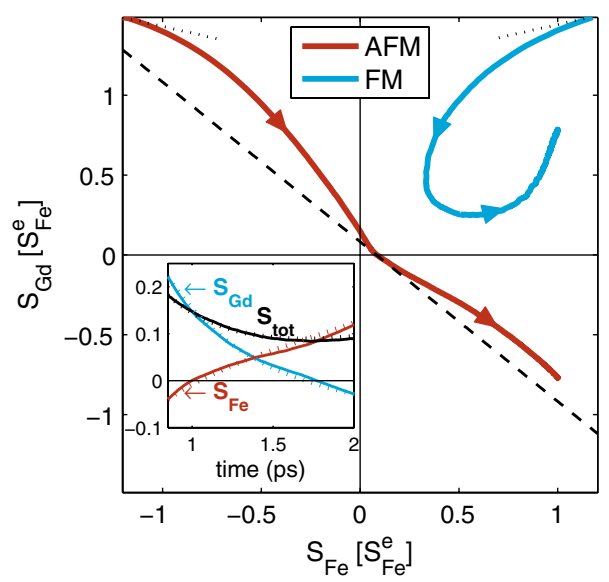

FIG. 2 (color online). Atomistic spin dynamics simulation of laser-induced spin dynamics of a model GdFe system with either antiferromagnetic (AFM) or ferromagnetic (FM) sublattice coupling. The spin dynamics of $\mathrm{Gd}$ is shown as function of the spin dynamics of Fe, both scaled with the final value of Fe $S_{\mathrm{Fe}}^{e}$. For both types of coupling the sublattices demagnetize at distinct time scales, where the dotted line indicates the expectation from Eq. (4). The dashed line shows a trajectory that would be obtained with purely exchange relaxation. Only with AFM coupling the sublattices do reverse, showing temporal FM alignment on the ps time scale, as highlighted in the inset, where dotted lines indicate a fit with the phenomenological model.

temperature is initially $T_{0}=200 \mathrm{~K}$, it peaks at about $T_{P}=$ $1400 \mathrm{~K}$ and reaches finally $T_{F}=400 \mathrm{~K}$. We visualize the results in Fig. 2 by showing the evolution of $S_{\mathrm{Gd}}$ as function of $S_{\mathrm{Fe}}$, where both are normalized to the final value of $\mathrm{Fe}$ $S_{\mathrm{Fe}}^{e}$. Initially (top left), when the temperature is high, $S_{\mathrm{Fe}}$ demagnetizes faster than $S_{\mathrm{Gd}}$. The dotted line indicates the estimate determined by Eq. (4) showing reasonable agreement given that the peak temperature is only $T_{P} \approx 2 T_{C}$. Subsequently, the spin of $\mathrm{Fe}$ reaches zero first and becomes temporarily parallel with the spin of Gd until also the spin of Gd reverses. This is further highlighted by the inset of Fig. 2, which presents the evolution of the sublattices as a function of time. In this time interval we can fit the ASD results (solid lines) quite well with our models introduced above (dotted lines) for the parameters $A / B=4, J_{12} / B=$ $-1, \bar{S}_{2}=1, \lambda_{1}=\lambda_{2}=0.5 \lambda_{e}$ and the time scaled with $\left(B \lambda_{e}\right)^{-1}=1.917 \mathrm{ps}$. Note that the sign of $S_{\mathrm{Gd}}+S_{\mathrm{Fe}}$ remains positive on the picosecond time scale, where $T<T_{C}$, indicating that exchange is dominating. This is also confirmed by the dashed line in the phase plot, which shows the trajectory that would be obtained by only exchange relaxation. The relaxation to the new equilibrium values proceeds upon further cooling of both sublattices. Note that in principle also a fitting of the complete trajectory is possible when the temperature dependence of the parameters in the Landau expansion is known. By varying the laser intensity we found that the sublattice reversal only occurs above a critical fluence, as expected from the criterion in Eq. (5). For comparison, we also performed ASD simulations for the same model system but with FM coupling between the sublattices. For this case, the phase trajectory is located in one quadrant of the plane (top right of Fig. 2). Like in the AFM case, the sublattices show distinct dynamics but no reversal takes place, in accordance with Eq. (6) which only yields reversal for a temperature decrease. Hence, the simulations further confirm the predictive power of our phenomenological theory.

In summary, we propose a general theoretical framework for ultrafast spin dynamics in multisublattice magnets on the time scale of the exchange interaction. The dynamics can be classified in three regimes: First, a temperature dominated regime, below $1 \mathrm{ps}$, where relativistic relaxation dominates which yields distinct dynamics for sublattices with different magnetic moments. Second, on the ps time scale exchange relaxation is dominant, transferring angular momentum between the sublattices. Third, we predict that close to the critical temperature, where both relaxation parameters are of importance, exchange relaxation in sublattices with AFM coupling accelerates the demagnetization of both sublattices, while for FM coupled sublattices relativistic and exchange relaxation may counteract.

This research has received funding from the Nederlandse Organisatie voor Wetenschappelijk Onderzoek (NWO), EC FP7 [grants NMP3-SL-2008214469 (Ultramagnetron) and 214810 (FANTOMAS)], NASU (grant 228-11), STCU (grant 5210), VR, the KAW foundation and the ERC [grants 257280 (Femtomagnetism) and 247062 (ASD)]. The phase trajectories were made using the CurvesGraphics6 MATHEMATICA package by Gianluca Gorni [23] and the ArrowHead MATLAB package by Florian Knorn [24].

*J.Mentink@science.ru.nl

[1] A. Kirilyuk, A. V. Kimel, and T. Rasing, Rev. Mod. Phys. 82, 2731 (2010).

[2] E. Beaurepaire, J.-C. Merle, A. Daunois, and J.-Y. Bigot, Phys. Rev. Lett. 76, 4250 (1996).

[3] C. D. Stanciu et al., Phys. Rev. Lett. 99, 047601 (2007); K. Vahaplar et al., Phys. Rev. Lett. 103, 117201 (2009).

[4] A. V. Kimel et al., Nature (London) 429, 850 (2004); F. Hansteen, A. Kimel, A. Kirilyuk, and T. Rasing, Phys. Rev. B 73, 014421 (2006); F. Atoneche et al., ibid. 81, 214440 (2010).

[5] G. Ju et al., Phys. Rev. Lett. 93, 197403 (2004); J. Thiele, M. Buess, and C.H. Back, Appl. Phys. Lett. 85, 2857 (2004).

[6] L. D. Landau and E. M. Lifshitz, Phys. Z. Sowjetunion 8, 153 (1935).

[7] G. P. Zhang and W. Hübner, Phys. Rev. Lett. 85, 3025 (2000).

[8] B. Koopmans, J. J.M. Ruigrok, F. DallaLonga, and W. J. M. de Jonge, Phys. Rev. Lett. 95, 267207 (2005).

[9] U. Atxitia et al., Appl. Phys. Lett. 91 (2007). 
[10] N. Kazantseva et al., Europhys. Lett. 81, 27004 (2008).

[11] C. Stamm et al., Nature Mater. 6, 740 (2007).

[12] C. La-O-Vorakiat et al., Phys. Rev. Lett. 103, 257402 (2009).

[13] I. Radu et al., Nature (London) 472, 205 (2011).

[14] C. Schneider (2011), MISM, book of abstracts.

[15] S. I. Anisimov, B. L. Kapeliovich, and T. L. Perelman, Zh. Eksp. Teor. Fiz. 66, 776 (1974) [Sov. Phys. JETP 39, 375 (1974)].

[16] V. G. Baryakhtar, Zh. Eksp. Teor. Fiz. 87, 1501 (1984); 94, 196 (1988); Fiz. Nizk. Temp. 11, 1198 (1985).[Sov. Phys. JETP 60, 863 (1984); Sov. Phys. JETP 67, 757 (1988); Sov. J. Low Temp. Phys. 11, 662 (1985)].

[17] D. A. Garanin, Phys. Rev. B 55, 3050 (1997).
[18] W. F. Brown, Phys. Rev. 130, 1677 (1963); R. Kubo and N. Hashitsume, Prog. Theor. Phys. Suppl. 46, 210 (1970).

[19] T. Ostler et al. [Nature Commun. (to be published)].

[20] O. Tegus, E. Brück, K. H. J. Buschow, and F. R. de Boer, Nature (London) 415, 150 (2002).

[21] B. Skubic, J. Hellsvik, L. Nordström, and O. Eriksson, J. Phys. Condens. Matter 20, 315203 (2008); http:// www.physics.uu.se/en/page/UppASD.

[22] J. H. Mentink et al., J. Phys. Condens. Matter 22, 176001 (2010).

[23] http://users.dimi.uniud.it/ gianluca.gorni/.

[24] http://www.mathworks.com/matlabcentral/fileexchange/ 4538-arrowhead. 